\title{
Avaliação do Interesse na Carreira Acadêmica dos Médicos em Especialização em Anestesiologia
}

\author{
Gustavo Henrique Meurer ${ }^{1}$, Henrique Kozuki ${ }^{1}$, Getúlio R de Oliveira Filho, TSA ${ }^{2}$
}

\begin{abstract}
Resumo: Meurer GH, Kozuki H, Oliveira Filho GR - Avaliação do Interesse na Carreira Acadêmica dos Médicos em Especialização em Anestesiologia.

Justificativa e objetivos: Tem-se observado certa tendência à perda pelo interesse acadêmico nas diversas áreas da Medicina, inclusive Anestesiologia. O objetivo deste estudo foi pesquisar o interesse de médicos em especialização (ME) em Anestesiologia em seguir uma carreira acadêmica, bem como identificar os fatores determinantes dessa escolha.

Método: O estudo foi prospectivo e transversal. Selecionou-se uma amostra probabilística simples de médicos em especialização em Anestesiologia. Um questionário foi enviado a cada participante. As respostas foram submetidas a análises uni e multivariadas para determinar a frequência de predisposição acadêmica na amostra e determinação dos fatores preditivos independentes de predisposição acadêmica.
\end{abstract}

Resultados: Foram analisados 155 questionários (taxa de resposta $=38,7 \%$ ). Sessenta e nove participantes $(44,5 \%)$ manifestaram interesse em seguir uma carreira acadêmica. A análise multivariada identificou como fatores independentes de previsão da predisposição acadêmica: estar cursando o primeiro ano do curso de especialização (razão de chances $[R C]=2,52$; intervalo de $95 \%$ de confiança [IC 95\%] $=1,19-5,38$ ), ter apresentado trabalho científico em evento médico $(R C=3,78$; IC $95 \%=1,84-7,78)$ e pertencer à região sudeste $(R C=2,66$; IC $95 \%=1,31-5,39)$.

Conclusões: Uma parcela significativa dos ME brasileiros demonstrou interesse em seguir carreira acadêmica após o término do curso de especialização. Em comparação com a probabilidade de não manifestar predisposição acadêmica, os médicos do primeiro ano do curso de especialização apresentam chances 2,5 vezes maiores de se manifestarem interessados em uma carreira acadêmica; apresentação de trabalho científico em eventos médicos aumentou essa chance em 3,78 vezes; pertencer a CETs da região sudeste aumentou em 2,66 vezes a chance manifestar interesse acadêmico.

Unitermos: ANESTESIOLOGIA: educação.

[Rev Bras Anestesiol 2010;60(6): 567-576] OElsevier Editora Ltda.

\section{INTRODUÇÃO}

Em estudos realizados com populações de médicos residentes ou especialistas de diversas áreas de Medicina, inclusive Anestesiologia, tem-se observado uma tendência universal à perda progressiva do interesse por carreiras acadêmicas. Burocracia, baixa remuneração, falta de mentores ou de centros de pesquisa estruturados são apontados como principais fatores responsáveis por esse fenômeno ${ }^{1-7}$.

A academia se caracteriza pela produção de conhecimento. Acadêmicos são indivíduos que produzem o conhecimento de suas respectivas áreas de especialidade. No Brasil, o incentivo à atividade acadêmica tem sido reputado como pequeno ou inexistente ${ }^{8}$. Prova disso é a ainda modesta contribuição brasileira para o montante de publicações médicas, representada por $1,9 \%$ das publicações internacionais ${ }^{9} \mathrm{e}$ por $0,38 \%$ das publicações internacionais na área da Anestesiologia ${ }^{10}$. Por isso,

Recebido do CET/SBA Integrado de Anestesiologia da SES-SC, Hospital Governador Celso Ramos, Florianópolis, SC, Brasil.

1. M3 do CET/SBA Integrado de Anestesiologia da SES-SC, Florianópolis, SC

2. Doutor, em Anestesiologia, Responsável, pelo CET/SBA Integrado de Anestesiologia da SES-SC, Florianópolis, SC

Submetido em 5 de fevereiro de 2010.

Aprovado para publicação em 21 de junho de 2010.

Correspondência para:

Dr. Gustavo Henrique Meurer

CET/SBA Integrado de Anestesiologia da SES-SC, Hospital Governador Celso Ramos

Rua Irmã Benwarda 297, $3^{\circ}$ andar

88015-360 - Florianópolis, SC

E-mail: ghmeurer@hotmail.com parece conveniente que mais indivíduos ingressem na vida acadêmica, como uma forma de projetar o país no âmbito da comunidade científica global. Essa preocupação tem sido o foco de discussões sobre o tema em âmbito nacional.

Diversos fatores predisponentes e limitantes podem interferir nas escolhas dos médicos em especialização quanto ao destino que darão às suas carreiras após a conclusão da residência médica.

Conhecer estes fatores é, portanto, de fundamental importância para os educadores e outros membros da comunidade científica, interessados em promover o desenvolvimento científico nacional. Dado o ambiente pouco propício à carreira acadêmica ${ }^{8}$, a hipótese deste estudo foi de que apenas uma minoria dos médicos residentes de Anestesiologia manifestaria propensão em seguir uma carreira voltada à pesquisa e à educação após a conclusão da pós-graduação lato sensu.

Este estudo teve por objetivo avaliar quantitativamente o interesse dos médicos residentes em seguir uma carreira acadêmica em Anestesiologia e identificar os fatores preditivos dessa escolha.

\section{MÉTODO}

O estudo contou com a aprovação do Comitê de Ética em Pesquisa do Hospital Governador Celso Ramos, tendo como características ser prospectivo, transversal, voluntário e anônimo. Foi incluída no estudo uma amostra probabilística simples de médicos em especialização em Anestesiologia de ambos os sexos, dos primeiro, segundo e terceiro anos 
de especialização, pertencentes a Centros de Ensino e Treinamento (CETs) credenciados pela Sociedade Brasileira de Anestesiologia, no ano de 2008. Para cada participante da pesquisa, foram enviados pela internet um questionário e um termo de consentimento livre esclarecido através dos respectivos endereços eletrônicos cadastrados no portal da SBA. O questionário visava coletar as seguintes informações: ano no programa de especialização (ME1, ME2 e ME3), idade, gênero (masculino/feminino), região geográfica (norte, nordeste, centro-oeste, sudeste e sul), participação em trabalhos científicos (sim, não), percepção sobre o incentivo à pesquisa em seu CET (frequentemente, esporadicamente, raramente ou nunca) e interesse na carreira acadêmica (sim, não). Caso houvesse participado de projeto de pesquisa, era solicitado a informar a(s) fase (s) do estudo das quais participara (projeto, coleta de dados, análise estatística, redação do artigo, apresentação em evento médico). Havendo interesse em seguir uma carreira acadêmica, solicitavam-se informações sobre as áreas de interesse acadêmico (ensino, pesquisa, ensino e pesquisa, incerto), titulação acadêmica pretendida (especialização, mestrado, doutorado, pós-doutorado) e os principais obstáculos a serem enfrentados para obtê-la (burocracia, retorno financeiro, jornada de trabalho, requisitos para publicações, recursos físicos, supervisão). Não havendo interesse, o participante deveria manifestar-se nesse sentido ou o momento de sua formação em que o perdeu, declarando os principais motivos que o levaram a essa tomada de decisão (burocracia, retorno financeiro, jornada de trabalho, requisitos para publicações, recursos físicos, supervisão etc.).

Para o cálculo do tamanho da amostra, estimou-se que o percentual de participantes que demonstrariam interesse em seguir uma carreira acadêmica seria em torno de $10 \%$, ou um terço do percentual de residentes com propensão acadêmica em estudos realizados em países desenvolvidos, onde o percentual fica em torno de $30 \%{ }^{6}$. Considerando um nível de confiança de $95 \%$ e uma taxa de resposta estimada de $30 \%{ }^{11,12}$, estimou-se que 480 médicos em especialização teriam de ser convidados a participar do estudo.

Foram enviadas mensagens eletrônicas com o questionário a 480 médicos em especialização, cujos endereços eletrônicos foram selecionados através de números aleatórios gerados eletronicamente e atribuídos aos 1.065 endereços eletrônicos de membros aspirantes disponíveis no portal da SBA. Aos participantes que não responderam ao primeiro envio, o questionário foi reenviado por mais duas vezes, com intervalos mensais.

Os dados obtidos foram submetidos às seguintes análises estatísticas (SPSS v.12, SPSS Inc., Chicago): estatística descritiva e análise de consistência interna do questionário (coeficiente alfa de Cronbach). Os dados foram submetidos a análises univariadas através dos testes exatos de Fisher, Quiquadrado e $t$ de Student. As variáveis significativas nas comparações entre os participantes que manifestaram predisposição ou não em seguir uma carreira acadêmica entraram na construção de um modelo de regressão logística para identificação dos fatores independentes de previsão da predisposição do ME (médico em especialização) em seguir carreira acadêmica.
O nível de significância aceito foi inferior a 5\% de probabilidade de erro estatístico tipo I.

\section{RESULTADOS}

Dos 480 e-mails enviados, 46 apresentaram falhas no envio e foram descartados, sendo respondidos 168 e-mails de um total de 434 , o que gerou uma taxa de retorno de $38,7 \%$. Dentre os e-mails respondidos, 11 foram desconsiderados devido a erros de preenchimento não sendo incluídos na análise do trabalho, restando, ao final um $n$ de 155 participantes o que está de acordo com o cálculo amostral prévio. O coeficiente alfa de Cronbach do questionário foi igual a 0,7. As características demográficas dos participantes do estudo se encontram na Tabela I. Os percentuais de resposta aos itens do questionário são mostrados nas Tabelas II, III e IV.

Tabela I - Dados Demográficos

\begin{tabular}{lll}
\hline & $\mathrm{n}$ & $\%$ \\
\hline Sexo * & 87 & 56 \\
$\quad$ Masculino & 67 & 44 \\
Feminino & $27,33 \pm 2,23$ & \\
$\quad$ Idade (anos) ** & & \\
Ano de especialização * & 49 & 32 \\
ME1 & 52 & 33 \\
ME2 & 54 & 35 \\
ME3 & & \\
Região * & 84 & 54 \\
Sudeste & 27 & 17 \\
Sul & 23 & 15 \\
Centro-Oeste & 18 & 12 \\
Nordeste & 3 & 2 \\
Norte &
\end{tabular}

* Variáveis categóricas expressas como frequência (n) e respectivas percentagens (\%);

** Variável contínua expressa como média e desvio-padrão e seus respectivos extremos.

Tabela II - Estatísticas Descritivas das Perguntas Respondidas por Todos os Participantes

\begin{tabular}{lll}
\hline & $\mathrm{n}$ & $\%$ \\
\hline Participação em trabalhos científicos & 115 & 74,2 \\
Sim & 38 & 24,5 \\
Não & 2 & 1,3 \\
Não responderam & & \\
Fases da pesquisa em que atuaram os indivíduos que participaram \\
de trabalhos científicos* \\
Projeto & 86 & 74,8 \\
Coleta de dados & 99 & 86,1 \\
Análise estatística & 63 & 54,8 \\
Redação do artigo & 82 & 71,3 \\
Apresentação em evento médico & 72 & 62,6 \\
Autoria de trabalho publicado* & & \\
Não & 81 & 52,2 \\
Sim, publicação nacional & 56 & 36,1 \\
Sim, publicação internacional & 18 & 11,6 \\
Incentivo à produção científica durante a residência & \\
Frequentemente & 49 & 31,6 \\
Esporadicamente & 55 & 35,5 \\
Raramente & 40 & 25,8 \\
Nunca & 11 & 7,1 \\
\hline
\end{tabular}

* Questão admitiu seleção de mais de uma opção; por essa razão, a soma dos números de respostas em cada pergunta pode não coincidir com o número total de participantes do estudo. 
Tabela III - Estatísticas Descritivas das Perguntas Respondidas apenas pelos Participantes com Interesse em uma Carreira Acadêmica $(n=69)$

\begin{tabular}{lll}
\hline & $\mathrm{n}$ & $\%$ \\
Área de atuação & 33 & 47,8 \\
Ensino & 31 & 44.9 \\
Ensino e pesquisa & 3 & 4,3 \\
Pesquisa & 1 & 1,4 \\
Incerto & 1 & 1,4 \\
$\quad$ Não responderam & \\
Principais obstáculos à produção científica* & \\
Burocracia & 20 & 29 \\
Retorno financeiro & 22 & 31,9 \\
Jornada de trabalho & 32 & 46,4 \\
Requisitos para publicações & 7 & 10,1 \\
Recursos físicos & 13 & 18,8 \\
Supervisão & 13 & 18,8 \\
Nível de instrução pretendido & & \\
Especialização apenas & 8 & 11,6 \\
Mestrado & 11 & 15,9 \\
Doutorado & 35 & 50,7 \\
Pós-Doutorado & 12 & 17,4 \\
Não responderam & 3 & 4,3 \\
\hline
\end{tabular}

* Questão admitiu seleção de mais de uma opção; por essa razão, a soma dos números de respostas em cada pergunta pode não coincidir com o número total de participantes do estudo.
Tabela IV - Estatísticas Descritivas das Perguntas Respondidas apenas pelos Participantes sem Interesse em uma Carreira Acadêmica $(n=86)$

\begin{tabular}{lll}
\hline Questão & $\mathrm{n}$ & $\%$ \\
\hline Período em que ocorreu a perda de interesse & pela vida acadêmica \\
Nunca existiu & 29 & $33,7 \%$ \\
Perdeu-se durante a faculdade & 25 & $29,1 \%$ \\
Perdeu-se durante o ME1 & 19 & $22,1 \%$ \\
Perdeu-se durante o ME2 & 5 & $5,8 \%$ \\
Perdeu-se durante o ME3 & 5 & $5,8 \%$ \\
Não responderam & 3 & $3,5 \%$ \\
Principal motivo que contribuiu para a perda de interesse pela vida \\
acadêmica * \\
Burocracia & 28 & $32,6 \%$ \\
Retorno financeiro & 26 & $30,2 \%$ \\
Jornada de trabalho & 32 & $37,2 \%$ \\
Requisitos para publicações & 6 & $7 \%$ \\
Recursos físicos & 6 & $7 \%$ \\
Falta de supervisão & 18 & $20,9 \%$ \\
\hline
\end{tabular}

* Questão admitiu seleção de mais de uma opção; por essa razão, a soma dos números de respostas em cada pergunta pode não coincidir com o número total de participantes do estudo.

Tabela V - Comparações entre os Participantes com e sem Interesse em seguir uma Carreira Acadêmica quanto às Variáveis do Estudo e suas Respectivas Categorias

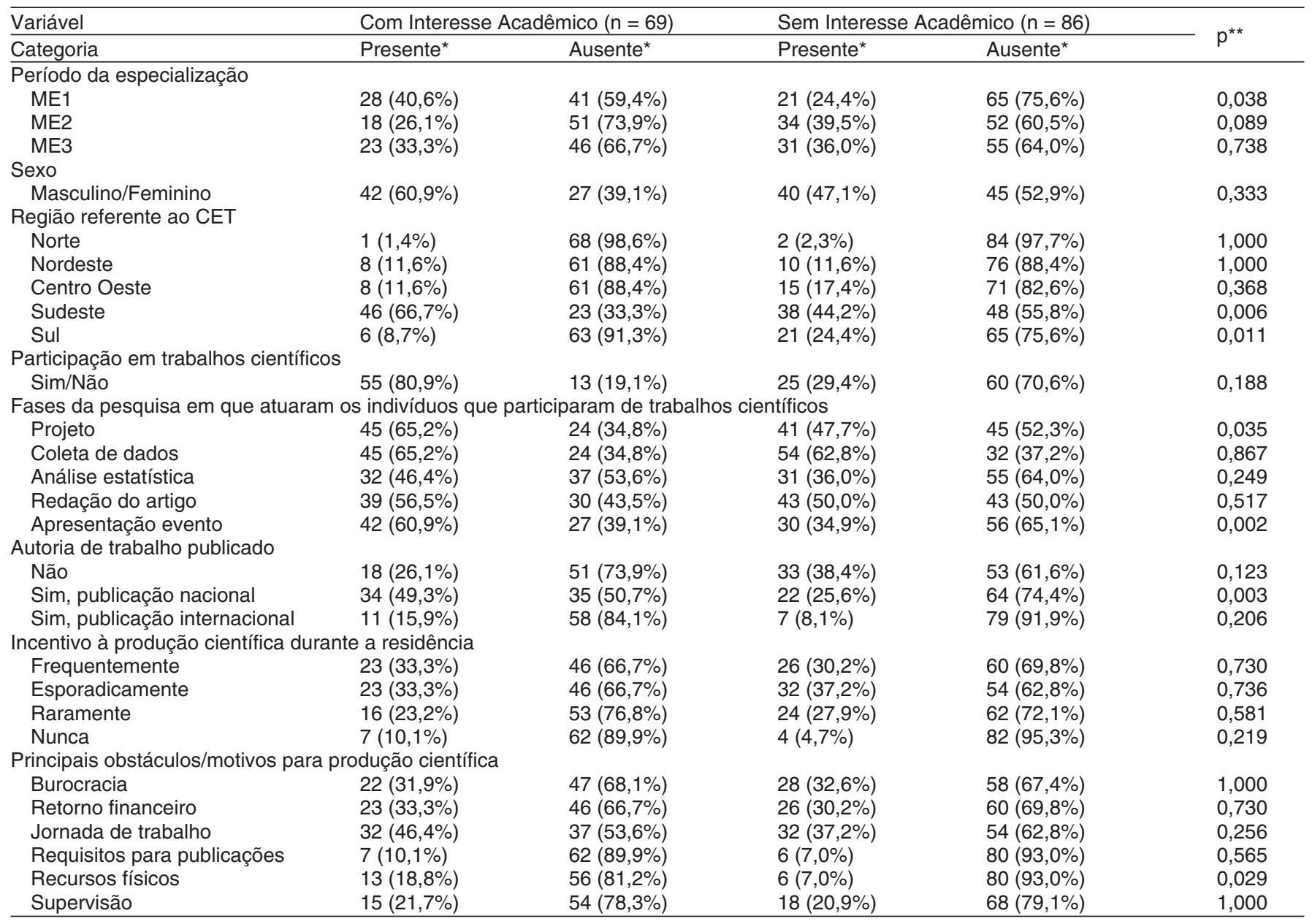

* Valores expressos em frequência (percentuais), extraídos de tabelas de contingência 2 x 2 utilizadas para a realização dos testes exatos de Fisher.

** $O$ valor de $p$ refere-se às comparações descritas acima por testes exatos de Fisher bicaudais. 
Sessenta e nove participantes $(44,5 \%)$ responderam afirmativamente à pergunta "Você tem interesse em seguir uma carreira acadêmica?", o que caracterizou o subgrupo de ME com predisposição em seguir uma carreira acadêmica. Considerando-se como variável dependente o médico em especialização ter ou não interesse na carreira acadêmica e sendo todas as outras consideradas variáveis independentes, quando submetidas à análise univariada foram significativas as seguintes: categoria "ME1" da variável "ano de especialização", categorias "sudeste" e "sul" da variável "região referente ao CET", categoria "projeto" e "apresentação em evento médico" da variável "fase da pesquisa em que atuaram", categoria "publicação nacional" da variável "autoria de trabalho publicado" e a categoria "recursos físicos" da variável "obstáculos à produção científica” (Tabela V).

A regressão logística identificou os seguintes fatores de previsão independentes para a predisposição em seguir uma carreira acadêmica: ser ME1 (razão de chances $(R C)=$ 2,52 ; intervalo de $95 \%$ de confiança $($ IC95\%) $=1,19$ $5,38)$, ter apresentado trabalho em evento médico $(\mathrm{RC}=$ 3,$78 ;$ IC95\% = 1,84 - 7,78) e pertencer a um CET da região sudeste $(R C=2,66$; IC95\% = 1,31 - 5,39). O percentual de classificação correta do modelo aplicado à amostra original foi de $69 \%$.

\section{DISCUSSÃO}

Uma parcela significativa dos residentes que participaram do estudo (44,5\%) demonstrou interesse em seguir carreira acadêmica. No final da década de 1980, Hillman e col. relataram prevalência de predisposição acadêmica de $28 \%$ em radiologistas e de $19 \%$ em médicos residentes de radiologia ${ }^{1}$. Em 2006, Reck e col. relataram prevalência de predisposição em seguir uma carreira acadêmica de $36,9 \%$ entre os residentes de Dermatologia e de $72,5 \%$ entre os aspirantes à residência na especialidade. Tomados em conjunto e considerando a possibilidade de viés de seleção, os resultados desses três estudos sugerem aumento da predisposição acadêmica entre os médicos residentes. A favor dessa hipótese, está o fato de que os itens do questionário utilizado no presente levantamento foram extraídos dos dois estudos anteriores supracitados, o que permite a realização de comparações qualitativas. Esse possível aumento do interesse acadêmico evidenciado no decorrer dos anos é compatível com o aumento significativo da contribuição nacional para a literatura científica internacional, que, embora pequena diante do total, coloca o país entre os quatro com maior crescimento na última década ${ }^{9}$.

Promover aumento sempre crescente pela carreira acadêmica deve ser o foco dos centros de formação, através da busca de fatores que possam incentivá-la. Segundo estudo de Lynch e col., realizado com dermatologistas acadêmicos, os dois fatores mais importantes na escolha de uma carreira acadêmica foram a admiração pelos professores-orientadores durante a residência e as experiências de ensino vivenciadas com os estudantes de Medicina ${ }^{4}$. Os fatores independentes encontrados no presente estudo foram: ter apresentado tra- balho em evento médico, pertencer a um CET localizado na região sudeste e cursar o primeiro ano de residência médica. Assim como observado por Hillman e col. ${ }^{1}$, encontrou-se maior inclinação acadêmica por residentes pertencentes a instituições mais bem classificadas pelas respectivas sociedades médicas. A região sudeste do Brasil concentra as maiores universidades e os centros de pesquisa do país. Estar em formação em um programa de residência médica localizado nessa região foi fator independente de previsão da predisposição em seguir uma carreira acadêmica. Além disso, é bem provável que, durante o processo de formação acadêmica na região sudeste, um indivíduo tenha maior chance de estar exposto ao convívio com anestesiologistas acadêmicos, os quais podem servir de modelo, guiando-os e estimulandoos. Lynch e col. observaram que a maioria dos residentes de Dermatologia participantes do estudo somente decidiu seguir a carreira acadêmica após o segundo ano de residência 4 . Neste estudo, a condição de residente de Anestesiologia de primeiro ano foi fator de previsão independente para a predisposição em seguir uma carreira na Anestesiologia acadêmica. Diferenças culturais, de especialidade ou de metodologia podem eventualmente explicar essa diferença entre o corrente estudo e o citado anteriormente ${ }^{4}$. Isso porque este trabalho tem caráter transversal e o fato de o ME1 estar ingressando na residência médica poderia funcionar como viés, já que poderia mostrar-se mais entusiasmado pela especialização e o meio acadêmico e, assim, estar mais propenso a manifestar interesse em seguir uma carreira acadêmica. Esse viés poderia ser contornado com um estudo futuro de caráter prospectivo, com o acompanhamento do ME durante os três anos da especialização.

Por outro lado, ter conhecimento dos fatores que podem desestimular o indivíduo em seguir uma carreira acadêmica e buscar minimizá-los faz parte de uma estratégia que busca fortalecer o meio acadêmico. Bergstresser ${ }^{13}$ citou, entre os obstáculos para seguir uma carreira acadêmica, questões financeiras, falta de controle sobre a carreira, necessidade de adotar uma postura política dentro da instituição e pouco tempo para o desempenho das exigências de produção acadêmica. No estudo realizado por Reck e col. ${ }^{6}$, os obstáculos identificados foram: burocracia, questão financeira, falta de supervisão e de orientação, escassez e inadequação de ambientes para a prática acadêmica. Hillman e col. ${ }^{1}$ enumeraram, em ordem decrescente de importância, os seguintes obstáculos: encontrar tempo para a prática acadêmica, pressões para a prática clínica, obtenção de recursos para a pesquisa, tempo insuficiente de treinamento em pesquisa, dificuldade em encontrar colegas bem instruídos, serviços de suporte insuficientes, obrigações pessoais e familiares, pressão para o ensino, dificuldade na obtenção de boas ideias para pesquisa e na respectiva publicação. No presente estudo, os principais obstáculos apontados pelos participantes na busca por uma carreira acadêmica foram jornada de trabalho, questões burocráticas relacionadas à pesquisa e retorno financeiro, entendido como uma desvalorização do academicismo em relação à prática privada. No entanto, nenhum desses fatores mostrou-se significativo quando submetidos à 
análise multivariada, não podendo ser considerados fatores de previsão independentes.

Assim como no estudo desenvolvido por Reck e col. ${ }^{6}$, a maioria dos participantes do presente estudo que manifestaram predisposição em seguir uma carreira acadêmica referiu o ensino como principal foco da atuação acadêmica. A academia caracteriza-se pela produção e a transmissão de conhecimento e respectiva publicação. O pouco interesse demonstrado pelos participantes com predisposição acadêmica em se dedicar à pesquisa merece consideração, sugerindo certa confusão em relação ao significado de uma carreira acadêmica.

As limitações do presente estudo se referem ao seu desenho, presentes em qualquer pesquisa que inclua coleta de dados por meio eletrônico: validação externa dos dados obtidos, obtenção de uma amostra representativa da população e uma taxa de resposta adequada ${ }^{11,12}$. Nesse tipo de estudo, além de as taxas de resposta normalmente serem baixas, é possível que ocorra viés de seleção, já que muitos participantes que responderam ao questionário poderiam ser justamente aqueles com maior propensão em seguir a carreira acadêmica. Outra limitação se deve ao fato de este estudo ter caráter transversal, não permitindo acompanhamento prospectivo dos residentes ao longo dos três anos do programa de residência médica.

Por fim, este estudo sugere que, para incentivar a formação de anestesiologistas acadêmicos, os programas de residência médica poderiam incentivar os médicos residentes, especialmente no primeiro ano de especialização, a realizar estudos científicos, com a respectiva apresentação em eventos científicos. Além disso, também se deve ressaltar a criação de estratégias para o desenvolvimento de habilidades referentes ao ensino durante o programa de residência médica, já que grande parte do interesse demonstrado pelos participantes se situa nessa área de atuação. É preciso contar não só com pesquisadores, mas também com professores para a formação do ambiente acadêmico. Os resultados deste estudo também sugerem que melhorias da produção científica e do ambiente educacional dos programas de residência médica poderiam estimular positivamente a predisposição dos residentes em seguir uma carreira acadêmica.

\section{REFERÊNCIAS / REFERENCES}

01. Hillman BJ, Fajardo LL, Witzke DB et al. - Factors influencing radiologists to choose research careers. Invest Radiol, 1989;24:842-848.

02. Hillman BJ, Fajardo LL, Witzke DB et al. - Influences affecting radiologists' choices of academic or private practice careers. Radiology, 1990;174:561-564.

03. Kuczkowski KM - To be or not to be (an academic anesthesiologist)? Acta Anaesthesiol Scand, 2005;49:127.

04. Lynch PJ, Harrell ER - Factors in the choice of an academic career. Results of a questionnaire. Arch Dermatol, 1971;103:328.
05. O'Sullivan PS, Niehaus B, Lockspeiser TM et al. - Becoming an academic doctor: perceptions of scholarly careers. Med Educ, 2009;43:335-341.

06. Reck SJ, Stratman EJ, Vogel C et al. - Assessment of residents' loss of interest in academic careers and identification of correctable factors. Arch Dermatol, 2006;142:855-858.

07 . Wyngaarden JB - The clinical investigator as an endangered species. N Engl J Med, 1979;301:1254-1259.

08. Schwartzman S, Balbachevsky E - A profissão acadêmica no Brasil, 1997. [Disponível em: http://www.schwartzman.org.br/simon/pdf/ prof_acad.pdf].

09. Karlberg JPE - Biomedical publication trends by geographic region. Clin Trial Magnifier, 2009;2:682-702

10. Figueredo E, Sanchez Perales G, Munoz Blanco F - International publishing in anaesthesia - how do different countries contribute? Acta Anaesthesiol Scand, 2003;47:378-382.

11. Braithwaite D, Emery J, De Lusignan S et al. - Using the Internet to conduct surveys of health professionals: a valid alternative? Fam Pract, 2003;20:545-551.

12. Oliveira Filho GR, Schonhorst $L$ - Attitudes of residents and anesthesiologists toward basic sciences. Anesth Analg, 2006;103:137-143.

13. Bergstresser PR - Perceptions of the academic environment: a national survey. J Am Acad Dermatol,1991;25:1092-1096.

Resumen: Meurer GH, Kozuki H, Oliveira Filho GR - Evaluación del Interés en la Carrera Académica de los Médicos en Especialización en Anestesiología.

Justificativa y objetivos: Se ha venido observando una cierta tendencia a la pérdida del interés académico en las diversas áreas de la Medicina, incluso en la Anestesiología. El objetivo de este estudio fue investigar el interés de los médicos en especialización (ME) en Anestesiología, para continuar una carrera académica, como también identificar los factores determinantes de esa elección.

Método: El estudio fue prospectivo y transversal. Se seleccionó una muestra probabilística sencilla de médicos en proceso de especialización en Anestesiología. Un cuestionario fue enviado a cada participante. Las respuestas se sometieron al análisis uni y multivariado para determinar la frecuencia de la predisposición académica en la muestra y determinar los factores predictivos independientes de predisposición académica.

Resultados: Fueron analizados 155 cuestionarios (tasa de respuesta $=38,7 \%$ ). Sesenta y nueve participantes (un $44,5 \%$ ), manifestaron interés en seguir una carrera académica. El análisis multivariado identificó como factores independientes de previsión de la predisposición académica: estar cursando el primer año del curso de especialización (razón de chances $[R C]=2,52$; intervalo de un 95\% de confianza [IC $95 \%]=1,19-5,38)$, haber presentado un trabajo científico en un evento médico $(R C=3,78$; IC $95 \%=1,84-7,78)$ y pertenecer a la región sudeste $(R C=2,66 ;$ IC $95 \%=1,31-5,39)$.

Conclusiones: Una parte significativa de los MEs brasileños demostró interés en seguir una carrera académica después del término del curso de especialización. En comparación con la probabilidad de no manifestar predisposición académica, los médicos del primer año del curso de especialización presentan chances 2,5 veces mayores de manifestarse interesados en una carrera académica. La presentación de un trabajo científico en eventos médicos aumentó esa chance en 3,78 veces. Pertenecer a CETs de la región sudeste aumentó en 2,66 veces el chance de manifestar interés académico. 